M. Hardmeier

S. Haller

A. Steck

P. Lyrer

S. Engelter

S. Renaud

\section{Vertebral artery dissection presenting with fifth cervical root (C5) radiculopathy}

Received: 4 February 2006

Received in revised form: 26 March 2006 Accepted: 28 March 2006

Published online: 11 April 2007

Sirs: Peripheral motor deficits in the upper limb are rare manifestations of vertebral artery dissection (VAD) [1-6]. In cervical spine MRI signs of VAD may remain unrecognised. The case reported here highlights the need to exclude VAD in otherwise unexplained radiculopathies of the upper cervical cord.

A white 52-year-old waiter presented with severe proximal weakness of the left arm. Three weeks prior to presentation he had sustained minor trauma with bruises to the right chest and ten days later developed left-sided neck pain without radiation to the arms. Three days before presen-

M. Hardmeier $(\square) \cdot$ A. Steck $\cdot$ P. Lyrer

S. Engelter $\cdot S$. Renaud

Dept. of Neurology

University Hospital Basel

Petersgraben 4

4031 Basel, Switzerland

Tel.: +41-61/265-4151

Fax: +41-61/265-4100

E-Mail: mhardmeier@uhbs.ch

S. Haller

Division of Neuroradiology

Dept. of Diagnostic

Radiology, University Hospital Basel

Basel, Switzerland tation he noticed difficulties at work when serving his guests, because he could neither elevate nor rotate the left shoulder. His past medical history was significant only for vaccinations (polio, hepatitis A and tetanus) three months before. He has no family history of neuromuscular diseases.

On admission severe weakness of the following muscles was observed on the left: deltoid (2/5), supraspinatus (2/5), infraspinatus $(2 / 5)$ and biceps (3/5). Biceps and brachioradialis reflexes were absent and no sensory deficit was detected. The remainder of the neurological and physical exam was unremarkable. A C5 radiculopathy was suspected on clinical grounds, probably due to a disc herniation. Since there was neither radiating pain nor a sensory deficit infectious radiculopathy and idiopathic plexus neuritis were considered in the differential diagnosis.

In the CSF, however, cell count and protein were within normal limits and the Lyme serology was negative. Cervical MRI (Fig. 1 A and $B$ ) showed no relevant discopathy, but revealed a dissection of the left vertebral artery from C7 to $\mathrm{C} 2$ indicated by an intramural haematoma (open and thin arrows) in close relationship to the nerve root (filled arrows). MRangiography (Fig. 1 C) demonstrated a narrowed lumen of the extracranial left vertebral artery. Neurosonography revealed enlarged vessel diameter and narrowed vessel lumen of the V2segment of the left vertebral artery without intimal flap or haemodynamic changes. Diagnosis of left subadventitial extracranial VAD compressing the $\mathrm{C} 5$ nerve root was made. Electromyography confirmed an isolated acute denervation process of the $\mathrm{C} 5$ innervated muscles of the left arm. Therapy with acetylsalicylic acid (100 mg) was started [7]. The weakness resolved within four weeks.

We found eight other published cases of VAD with severe weakness of $\mathrm{C} 5$ and $\mathrm{C} 6$ innervated muscles as the only presenting symptom [1-6]. Patients were young (mean age 35.4 years), the delay between initial pain and occurrence of weakness ranged from three to ten days (mean 7.2 days). Neck pain was reported in eight patients, local pain in the shoulder or arm in four. Weakness was most prominent in the deltoid muscle in all cases, slight numbness in the upper arm occurred in five. All had a full recovery after one to five months. None suffered from stroke or TIA during followup.

Cases of VAD presenting only with peripheral motor deficits may be underdiagnosed due to a more benign clinical course and the difficulties in establishing the diagnosis. MRI is the preferred technique for spine imaging, but subtle signs of VAD may be missed in the first few days, because the hyperintensity of the intramural haematoma on $\mathrm{T} 1$ weighted images is not visible until the formation of methaemoglobin $[8,9]$. Here, neurosonography may provide useful information about altered vertebrobasilar perfusion and nonatherosclerotic arterial wall alterations.

The close anatomical relationship between the vertebral artery in the intraforaminal part and the anterior motor part of the nerve roots suggests a compressive aetiology due to mural haematoma in the vessel wall $[1,2,5]$. Alternatively, VAD may compromise the perfusion in the vasa nervorum and result in nerve root ischaemia. The C5 root seems to be the most susceptible root. 
Fig. 1 Axial MRI at the level of $\mathrm{C5}, \mathrm{T} 1$ weighted sequence with fat and flow suppression (A) shows intramural haematoma of the left vertebral artery (open arrows) in close relationship to the nerve root (filled arrows). Coronal T1 weighted MRI with fat suppression (B) shows intramural haematoma (arrows) of the entire transforaminal V2-segment of the left vertebral artery ranging from $\mathrm{C} 7$ to $\mathrm{C}$. Contrast enhanced MR-angiography (C) shows marked longitudinal narrowing of the left vertebral artery lumen (arrows)
A
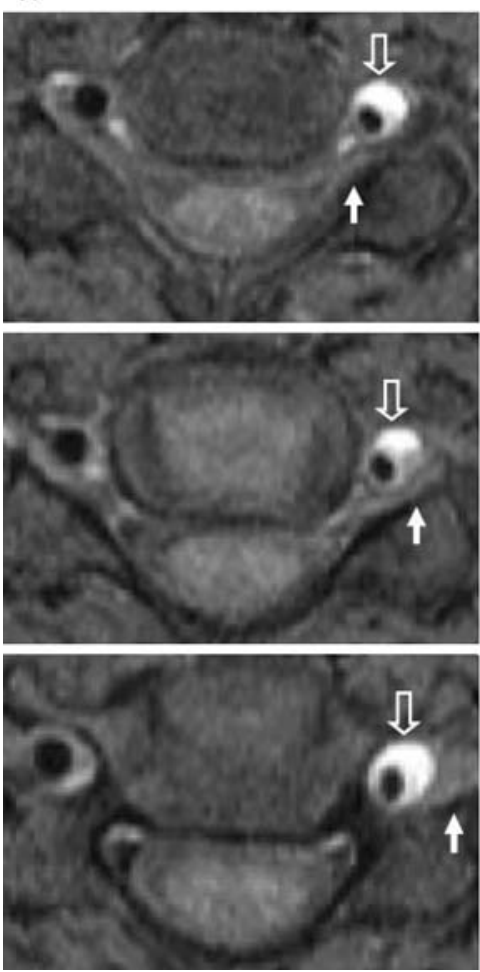

B

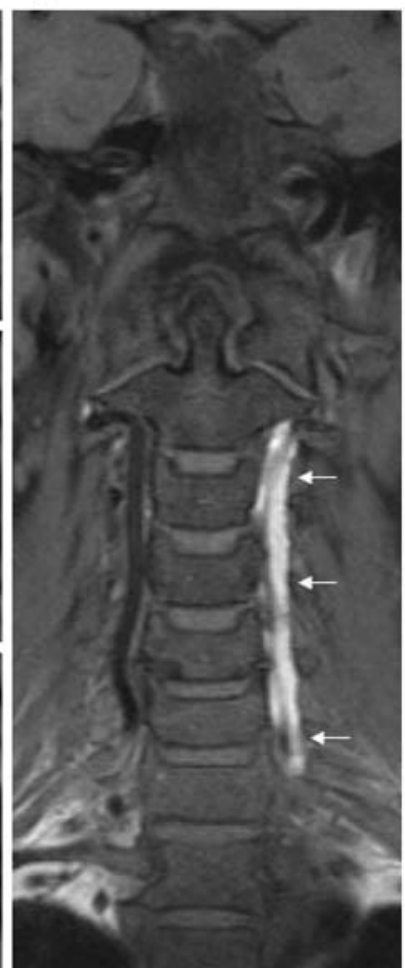

C

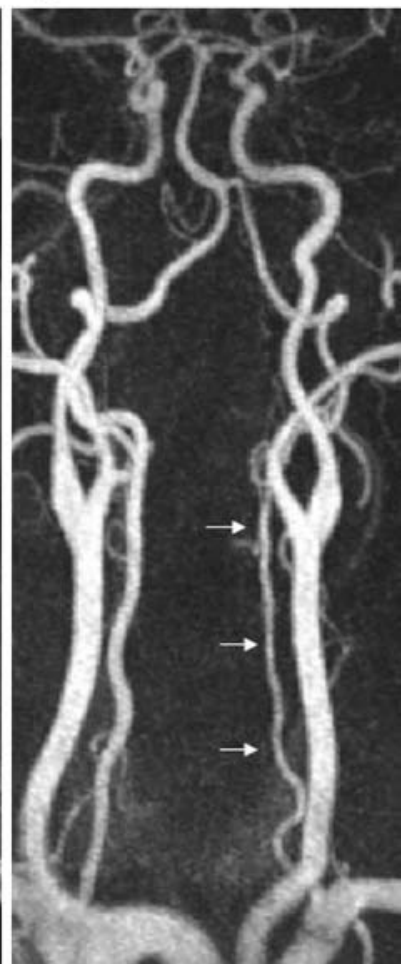

\section{References}

1. Dubard T, Pouchot J, Lamy C, Hier D, Caplan LR, Mas JL (1994) Upper limb peripheral motor deficits due to extracranial vertebral artery dissection. Cerebrovasc Dis 4:88-91

2. Hetzel A, Berger W, Schumacher M, Luecking $\mathrm{CH}$ (1996) Dissection of the vertebral artery with cervical root lesions. J Neurol 243:121-125

3. De Bray JM, Penisson-Besnier I, Giroud M, Klein J, Tanguy JY, Pasco A, Dubas F (1998) Peripheral motor deficit and three vertebral artery dissections. Rev Neurol (Paris) 154:762-766
4. Aggarwal A, Burton K (1999) Cervical root injury caused by vertebral artery dissection. J Clin Neurosci 2:166-168

5. Fournier JY, Amsler U, Weder B, Heilbronner R, Hildebrandt G (2000) Extracranial vertebral artery dissection causing cervical root lesion. J Neuroimaging 10:125-128

6. Crum B, Mokri B, Fulgham J (2000) Spinal manifestations of vertebral artery dissection. Neurology 55:304-306

7. Lyrer P, Engelter S (2003) Antithrombotic drugs for carotid artery dissection. Cochrane database Syst Rev 3:CD000255
8. Tay KW, U-King-Im JM, Trivedi RA, Higgins NJ, Cross JJ, Davies JR, Weissberg PL, Antoun NM, Gillard JH (2005) Imaging the vertebral artery. Eur Radiol 15:1329-1343

9. Paciaroni M, Caso V, Agnelli G (2005) Magnetic Resonance Imaging, Magnetic Resonance and Catheter Angiography for Diagnosis of Cervical Artery Dissection. In: Baumgartner RW, Bogousslavsky J, Caso V, Paciaroni (eds) Handbook on Cerebral Artery Dissection. Front Neurol Neurosci. Karger, Basel, vol 20, pp 102-118 